\title{
An underground three-dimensional displacement measurement system
}

\author{
Yifeng Cheng ${ }^{1}$, Qing $\mathrm{Li}^{2, \mathrm{a}}$, Nanying Shentu ${ }^{3}$ and Chao Zhang ${ }^{1}$ \\ 1. 2. 3 College of Mechanical and Electrical Engineering, China JiLiang University, Hangzhou 210018,China
}

\begin{abstract}
With the development of society, the damage of geological disaster to people's life and property is more serious, so the improvement of monitoring means of geological disaster is particularly urgent, there are some shortcomings in traditional monitoring methods, and it is impossible to measure the three-dimensional displacement of underground. The authors have designed an integrated three-dimensional displacement sensor to measure underground horizontal displacement and vertical displacement and tilt angle, and realize the real-time on-line monitoring of underground three-dimensional displacement visually through multi-group structure co-measurement.
\end{abstract}

\section{Introduction}

China's geological disasters are frequent, widely distributed, frequent and seriously harmful ${ }^{[1]}$, So the realtime on-line monitoring of geological hazards ${ }^{[2]}$ is particularly important, and the most direct monitoring means is to monitor the depth of the underground displacement situation. At present, the main monitoring methods are borehole inclinometer technology ${ }^{[3]}$, time domain reflection (TDR) testing technology ${ }^{[4]}$, although these methods are widely used, there are obvious shortcomings. First, borehole inclinometer technology needs many people to cooperate, the operations are complex ${ }^{[5]}$, and the soil movement makes the inclined tube multi-curved, so the inclinometer probe may not be put into $^{[6]}$. Second, the use of TDR technology monitoring means are difficult to monitor the size of the displacement [7] if the cable does not deform and destroy, and the local deformation of soft clay can not be effectively determined ${ }^{[8]}$. In view of the shortcomings of the above methods, the author designed an integrated sensor which can measure the three-dimensional displacement of the underground, combined with the communication module, the underground displacement can be monitored more intuitively.

\section{Overall scheme design}

Figure 1 is the general design of underground threedimensional displacement measurement system, wherein $\mathrm{C} 1, \mathrm{C} 2, \ldots, \mathrm{Cn}$ have the same structure, two adjacent sections form a group of displacement monitoring device. Multiple sets of devices are buried vertically in the rock and soil, when the displacement of the rock and soil, the distance between the integrated sensor changes, by measuring a set of integrated sensor mutual inductance voltage $\boldsymbol{U}_{\boldsymbol{o}}$ and the adjacent two sensor units of the distance $\mathbf{L}$ and the relative displacement direction and tilt angle $\boldsymbol{\theta}$, by comparing with the model which is established by experiments, the displacement data of rock and soil can be obtained, the sensor transmit the displacement data through the 485 bus $^{[9]}$ or wireless data network in real time via the host on the surface, the client can view the displacement data in real-time, so as to more accurately assess the displacement of the rock and soil, easy to develop disaster prevention and mitigation plan.

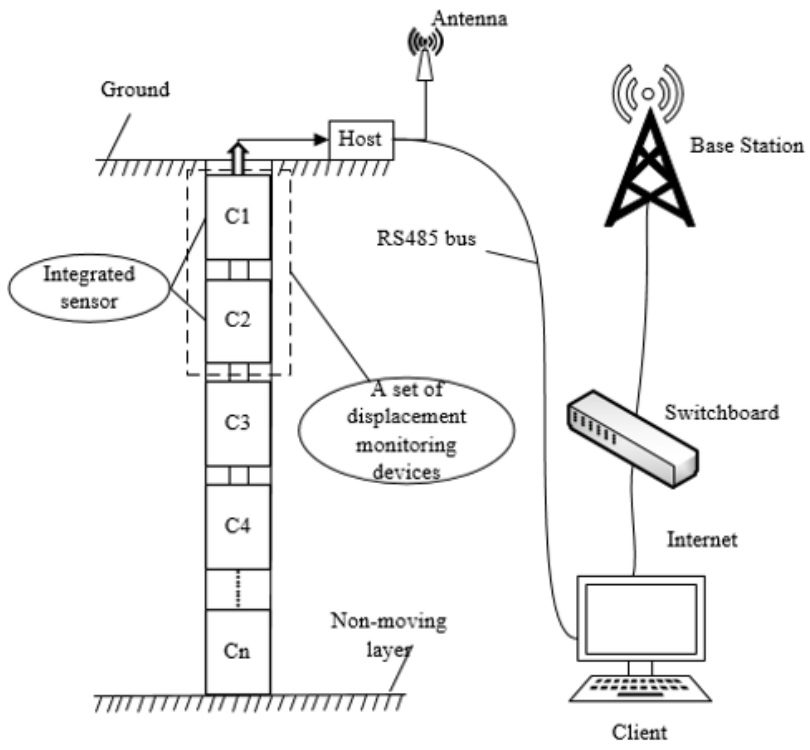

Figure 1. General design of underground three-dimensional displacement measurement system.

\section{Design of integrated sensor for underground three-dimensional displacement measurement}

a Corresponding author: lq13306532957@163.com 
The three-dimensional displacement integrated sensor consists of the measurement of mutual inductance voltage and the distance between adjacent two integrated sensor units measurement and displacement direction and tilt angle measurement between the adjacent two integrated sensor units, which is expressed as an example of a group of integrated sensors. Figure 2 is a set of integrated sensor structure diagram, in the figure $\mathrm{C} 1$ and $\mathrm{C} 2$ are identical units, each unit of the coil can be launched or received, when the $\mathrm{C} 2$ as a transmitting coil, $\mathrm{C} 1$ as a receiving coil for mutual inductance voltage measurement. The special metal wire is used to measure the distance between adjacent two integrated sensor units. One end of the special metal wire is fixed in the bottom of the $\mathrm{C} 1$, the remainder is completely pierced into the $\mathrm{C} 2$ in the $\mathrm{F} 1$ layer of the soft spiral tube coil, used to measure the line distance between the center of C1 bottom surface and the center of C2 the top surface, F2 layer for the mainboard, attitude measurement chip can be implemented accelerometer and gyroscope and electronic compass function, for measuring two units in each group of relative displacement direction and tilt angle, through the fixed time interval to send the measured data through the bus to the host which is located on the ground, through the calibration experiment with the model to calculate the horizontal and vertical displacement of each group of integrated adjacent sensors and the relative direction of movement and tilt angle, the host could get the three-dimensional displacement of the underground.

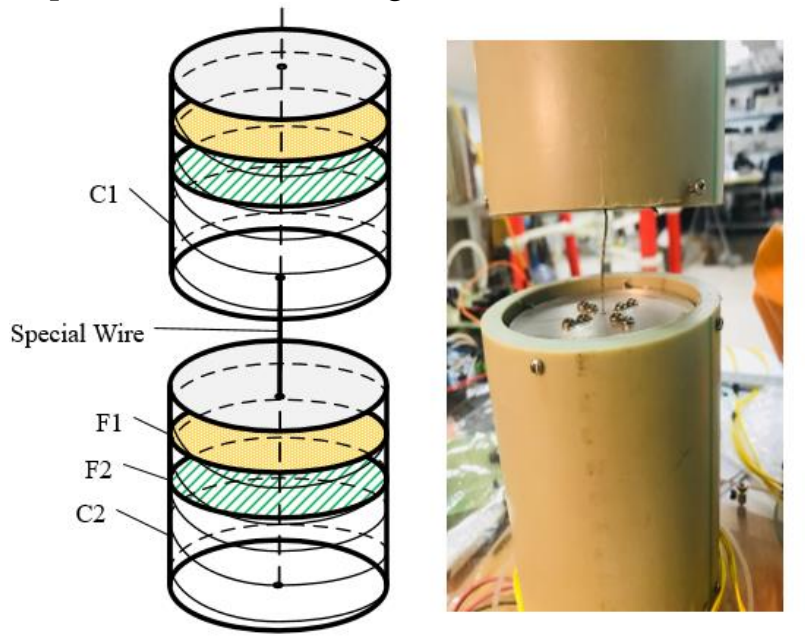

Figure 2. A set of integrated sensor structure diagrams.

\subsection{Principle of displacement measurement}

Figure 3 is the integrated sensor displacement measurement schematic diagram, $\boldsymbol{\theta}$ is two adjacent integrated sensor axis angle, can be obtained from the attitude measurement chip, $\mathbf{R}$ is the radius of the integrated sensor round tube, $\mathrm{L}$ is the straight distance between the center of the $\mathrm{C} 1$ Bottom surface and the center of the C2 top surface also the length of the wire drawn when the subsurface displacement occurs.

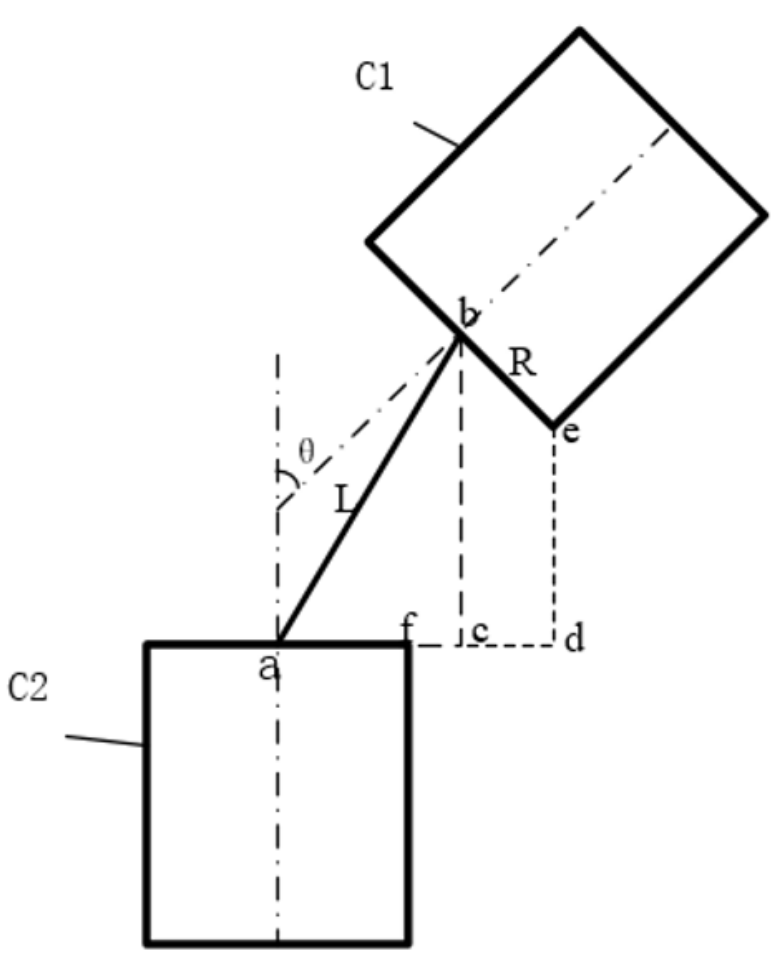

Figure 3. Integrated sensor displacement measurement schematic diagram.

Now another:

$$
\left\{\begin{array}{l}
a c=X \\
f d=x \\
b c=Y \\
e d=y \\
a b=L
\end{array}\right.
$$

They exist in the following mathematical relationships:

$$
\begin{aligned}
& Y=y+R \sin \theta \\
& X=x+R-R \cos \theta \\
& L=g(x, y, \theta)=\sqrt{X^{2}+Y^{2}}= \\
& \sqrt{(x+R-R \cos \theta)^{2}+(y+R \sin \theta)^{2}} \\
& Y=\sqrt{L^{2}-X^{2}}
\end{aligned}
$$

The mutual inductance voltage of the receiving coil is $\boldsymbol{U}_{\boldsymbol{o}}$ by the relative position $\mathbf{x}, \mathbf{y}$ and the relative axis angle $\boldsymbol{\theta}$ between two integrated sensor units in a set of measurement sensors.

Firstly, a mathematical model of $\boldsymbol{U}_{\boldsymbol{o}}=\boldsymbol{f}(\boldsymbol{L}, \boldsymbol{x}, \boldsymbol{\theta})$ or $\boldsymbol{U}_{\boldsymbol{o}}=\boldsymbol{f}(\boldsymbol{L}, \boldsymbol{y}, \boldsymbol{\theta})$ is established by recording the corresponding $\mathbf{x}, \mathbf{y}$ and $\boldsymbol{\theta}$ and $\mathrm{L}$ values of the specific 
receiving coil mutual inductance voltage $\boldsymbol{U}_{\boldsymbol{o}}$ through a large number of experiments.

When measuring, through the circuit measurement to obtain the length of $\mathrm{L}$ and angle $\boldsymbol{\theta}$ and the receiving coil mutual inductance voltage $\boldsymbol{U}_{\boldsymbol{o}}$, we can get a group of adjacent two integrated sensor units in the relative position, through the measurement of the displacement of a number of integrated sensors, the displacement of the overall sensor around the situation.

\subsection{Hardware design of integrated sensor for underground three-dimensional displacement measurement}

Figure 4 is the underground three-dimensional displacement measurement integrated sensor working flow chart, the use of STM32 combined with AD9851 chip using direct digital synthesis (DDS) ${ }^{[10]}$, generate amplitude frequency stable sine wave signal, through the DC-Block and amplifying circuit and power amplifier circuit output to the analog switch, the analog switch defaults to loading the signal into the lengthmeasuring bridge, when the displacement occurs the output voltage signal of the long bridge changed, amplifies the output signal after it is converted to the DC signal by a rectifier circuit then use the STM32 internal ADC to gather them, the length $\mathrm{L}$ of this time is obtained by comparing with the calibrated data, After measuring L, STM32 control analog switch switching, loading the output signal of the power amplifier circuit into the mutual inductance transmitting coil, the signal of the output of mutual inductance receiving coil is collected and recorded by the STM32 internal ADC after the rectifier circuit. At the same time, the attitude sensor sends the measured displacement direction and the tilt angle data into the STM32 via RS485 Bus then STM32 send the aggregated data to the host which on the surface.

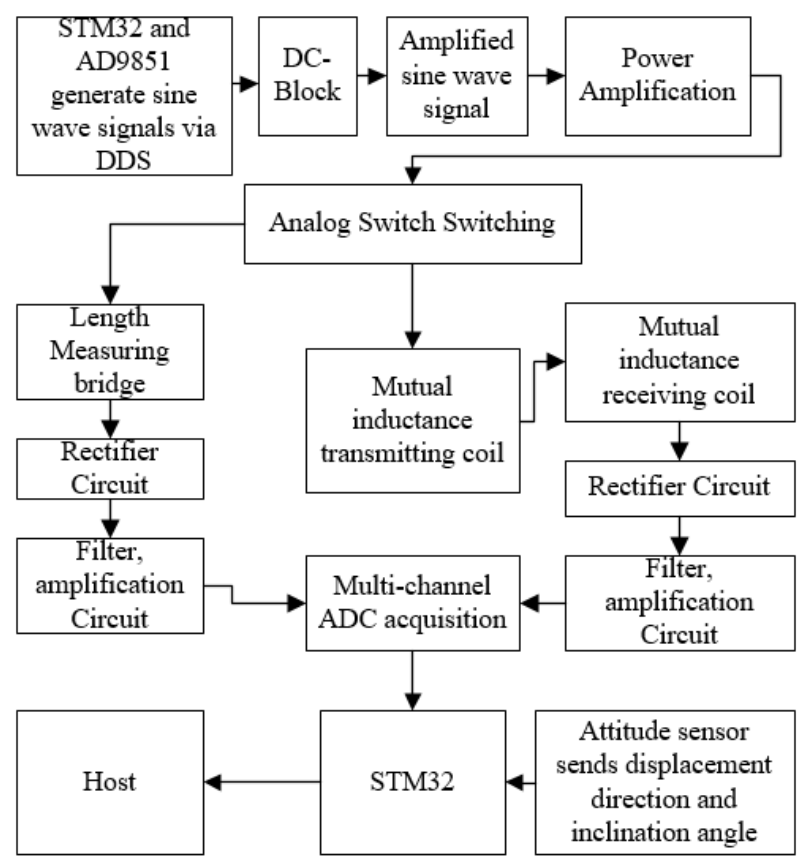

Figure 4. Underground three-dimensional displacement measurement integrated sensor working flow chart.

\subsection{Modeling experiments}

The displacement of a set of integrated sensors can be simulated by using the five-axis displacement table, the integrated sensor unit is completely symmetric about the central axis, one unit C2 is fixed on the table, simulating the movement of another unit $\mathrm{C} 1$ in one quadrant to represent other quadrants.

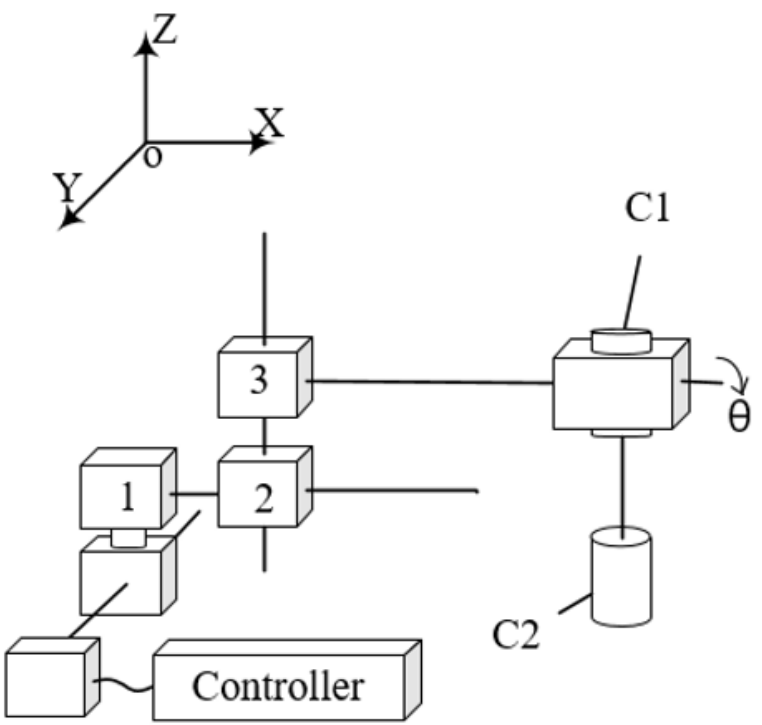

Figure 5. Motion diagram of five-axis displacement table

Figure 5 is the five-axis displacement table motion diagram, C1 and C2 form an integrated set of sensors, the $\mathrm{C} 2$ fixed on a flat table, $\mathrm{C} 1$ mounted at the end of the five-axis displacement stage, at the beginning, two 
integrated sensor unit axis coincident. The slider 1 can be moved along the y-axis, the slider 2 can move along the $\mathrm{z}$-axis, the slider 3 can move in the $\mathrm{x}$-axis direction, and the $\mathrm{C} 1$ can rotate the $\boldsymbol{\theta}$ angle along the arrow direction of the graph with the slider 3 horizontal axis. Several experiments recorded the slider 1 each movement distance namely $\mathrm{C} 1$ and $\mathrm{C} 2$ relative horizontal displacement $\mathbf{x}$, while recording C2 mutual inductance voltage $\boldsymbol{U}_{\text {out }}$, wire total extraction length $\mathrm{L}$ and unit relative tilt angle $\boldsymbol{\theta}$, combined with (4) and (5) formula through MATLAB mathematical tools to build $\boldsymbol{U}_{\text {out }}=\boldsymbol{g}(\boldsymbol{x}, \boldsymbol{y}, \boldsymbol{\theta})$ mathematical model, it also establishes the relation model $\boldsymbol{U}_{\text {out }}=\boldsymbol{f}(\boldsymbol{L}, \boldsymbol{X}, \boldsymbol{\theta})$ between the mutual inductance voltage and the vertical displacement $\mathrm{L}$ and the horizontal displacement and the angle.

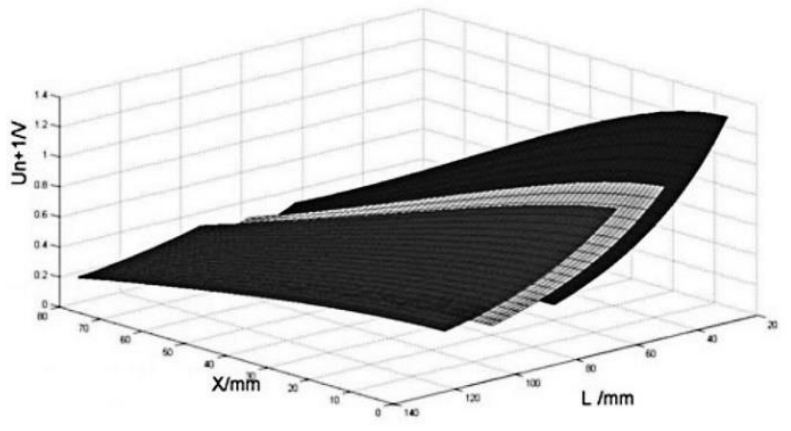

Figure 6. The relation model between the mutual inductance voltage $\mathrm{U}$ and the vertical displacement $\mathrm{L}$ and the horizontal displacement and angle

Fig. 6 is the $\boldsymbol{\theta}$ of $0^{\circ}$ (black), respectively, the relationship model of $25^{\circ}$ (light grey), $70^{\circ}$ (dark grey), when measured $\boldsymbol{U}_{\text {out }}, \boldsymbol{\theta}, \mathrm{L}$, can get the $\mathrm{X}$, and then according to Formula (5), can calculate $\mathrm{Y}$, with $\mathrm{X}$, Y and tilt angle and relative displacement direction angle, and the measurement data which from multiple units of measuring cells is sent to ground host to deal via RS485 bus, then the three-dimensional displacement of the underground soil body is obtained.

\section{Acknowledgments}

This work is funded by National Key research and Development project of China item No.2017YFC0804604, and National Natural Science Fund Project of China No. 61701467, and Key research and development projects in Zhejiang Province of China item No. 2018C03040.

\section{References}

1. Xiaojin. Research and development of integrated system for geological Disaster Emergency
Management J. Bulletin of Science and Technology, 2013,29 (4): 1.

2. $\mathrm{Xu}$ Zhenhong. Application of on-line monitoring technology in geological hazard monitoring $\mathbf{J}$. Geospatial information, 2016,14 (7): 93.

3. Zhang Chenjian, Zhang Xiaoshuai, Wen Dongming. The application of inclinometer in underground geological prospecting J. Shandong Coal Science and Technology, 2014,2:127.

4. Fu Jie. Research on landslide monitoring design based on TDR D. Shaanxi: Chang, 2014.

5. Ye Qiongyao of 59 landslide. Analysis of borehole inclinometer in nine-Wei landslide, J. Highway Traffic Technology (applied Technology), 2017 (3): 112.

6. Li Zheyun, Li Qing, Wang Yanjie. Underground deformation monitoring instrument system based on Hall effect J. Instrument technology, 2016, (4): 16.

7. Shi Yanxing, Zhang Qing. The application of TDR technology in the monitoring of Yaan-Xia-Kou landslide J. Survey of Science and Technology, 2005 (1): 55-57.

8. Pierce,C.E., Time Domain Reflectometry Measurements of Localized Soil Deformation[M]. $\mathrm{PhD}$ Thesis, Northwestern University, Evanston,Illinois, 1998:237.

9. Design of bus protocol intelligent initiation system based on RS485, Li, Liming (State Key Laboratory of Applied Physic-Chemistry Research, China); Lu, Qiao; Yin, Guofu Source: MATEC Web of Conferences, v 100, March 8, 2017, 13th Global Congress on Manufacturing and Management, GCMM 2016

10. Fully automated digital control system for Lab-onchip applications Morsy, Omar E. (Department of Electronics and Communication, Misr International University, Cairo, Egypt); Abo-Elela, Marwan; Ghallab, Yehya H; Hammad, Abdallah; Ismail, Yehea; El-Ghitani, Hassan Source: National Radio Science Conference, NRSC, Proceedings, v 2018March, p 369-376, May 3, 2018, Proceedings - 2018, 35th National Radio Science Conference, NRSC 2018 